\title{
Emergency Procurement Based on Supply Disruptions: Single-Sourcing or Dual-Sourcing
}

\author{
Xinjun $\mathrm{Li}^{*}$ and Xiaonan Zhang
}

School of Economic and Management, Yantai University, Yantai, Shandong, 264005, China

\begin{abstract}
Recent years have witness the pervasive supply disruptions and their impacts on supply chain performance. We investigate single or dual sourcing emergency model with partial supply disruptions. Introducing the concept of disruption proportion and delivery allocation, we first explore partial interrupted proportion of main supplier and delivery proportion of backup supplier. Under partial disruption, we then examine the expected profit of the manufacturer for single sourcing and multiple sourcing based on different disruption risk. Furthermore, we demonstrated the impact of delivery proportion between major supplier and backup supplier on emergency procurement strategy of the manufacturer. Finally we demonstrate the impact of delivery proportion between major supplier and backup supplier on manufacturer's emergency procurement strategy. Numerical examples show that the manufacturers should choose the best procurement strategy to maximize expected profit in different situations.
\end{abstract}

Keywords: Dual sourcing, emergency procurement, partial disruption, supply disruptions.

\section{INTRODUCTION}

With the development of lean production and economic globalization, supply chains become more and more complexity and vulnerable. Manufacturers tend to reduce the number of suppliers so as to increase economies of scale to achieve cost reduction and convenient management. Under this circumstance, once one of suppliers cannot delivery for disruption, it is possible bring the huge loss to downstream manufacturers or distributors and the entire supply chain. Facing with the supply disruption of the fire on March 2000 at the Philips plant in Albuquerque, NM, Nokia seek proactively other emergency suppliers, gaining great market share, and Ericsson waited passively, losing huge market share. On October 2011, Apple Mac Book Air's main supplier of metal chassis was ordered to suspend production for rectification by the local government because of environment destruction. These cases illustrate the necessary of dual-sourcing or backup suppliers.

There has been a growing stream of literature on supply disruptions. C. Blome [1] provides an review of the literature on supply chain disruption management and a detailed qualitative analysis. O. Tang and S. Musa [2] provide detailed presentation of the risk factors faced by suppliers, and provide recommendations for different procurement strategies. J. Burke and Janice E. Carrillo [3] examine shows that, unless the supplier's capacity is greater than the product demand, or the purchaser cannot obtain diversification benefits, single-source procurement dominant, otherwise dominant dual-source procurement. In Wang et al. [4], assuming two suppliers are unstable with different cost structures, the expected profit functions of four scenarios are established, using the approximation method to obtain the optimal ordering quantity. G. Yu et al. [5] argue that when supplier homogeneity degree is higher, dual-sourcing in response to supply disruptions have the advantage. Traditional multisourcing is conducive to promoting competition among suppliers, resulting to the lowest purchase price. K. Chen [6] study the manufacturers to retailers to adjust the price or give allowance to cope with Demand Disruptions. Fabian et al. [7] analyses the correlation between supply and demand uncertainty impacts on the optimal procurement strategy. X. Li et al. [8] explore dual-sourcing strategies to mitigate disruption risk, dividing disruptions into common cause failure and supplier-specific incident.

Our study is also related to the literature on backup suppliers. H. Jing [9] study on the advantages of backup supplier strategy and dual sourcing strategy. B. Tomlin [10] examine the dual sourcing flexibility and reliability using the newsboy model, and analyze the influence factors of secondary suppliers. WenJie Zhang [11] develop manufacturer's expected profits under different interruption probability. $\mathrm{Li}$ Xinjun et al. [12] present optimal ordering mechanisms based on options contracts for a triadic supply chain. Chen Junlin [13] research an unreliable supplier and a backup supplier of capacity constraints, the buyer how to choose the order strategy. Babich [14] study the influence factors of selecting backup supplier when supply disruptions.

Most of these documents focus on how to deal with some problems of supply and demand imbalance, with few articles about supply disruptions. In this paper, we explore the impact of the disruption proportion of major supplier and delivery proportion of backup supplier on the decision on manufacturer's emergency procurement strategy. In what follows, we will develop a set of manufacturer's expected profit of 
Table 1. Prices under different sourcing and corresponding demand function.

\begin{tabular}{|c|c|c|c|c|}
\hline & \multicolumn{2}{|c|}{$1-\gamma$} & \multicolumn{2}{|c|}{$\gamma$} \\
\hline & Prices & Market demand & Prices & Market demand \\
\hline Single sourcing & $c_{m}^{1}$ & $M-k c_{m}^{1}$ & $c_{m}^{1}$ & $M+\Delta M-k c_{m}^{1}$ \\
\hline Dual sourcing & $c_{m}^{2}, c_{b}^{1}$ & $M-k \cdot \frac{c_{m}^{2}+c_{b}^{1}}{2}$ & $c_{b}^{1}, c_{b}^{2}$ & $M+\Delta M-k \cdot \frac{c_{b}^{1}+c_{b}^{2}}{2}$ \\
\hline
\end{tabular}

single-sourcing and multiple-sourcing, respectively in the presence of supply chain disruption risks. We provide the best procurement strategy that the manufacturers should choose to maximize expected profit in different situations.

\section{MODEL ASSUMPTIONS AND SYMBOL DESCRIP- TION}

\subsection{Model Assumptions}

We consider a two-stage supply chain consisting of one manufacturer and two suppliers, where the wholesale prices offered by the two suppliers are different. The major supplier $(m)$ offers competitive wholesale price but lower reliability. The backup supplier (b) offers more reliable but more expensive.

Assuming supplier's capacity unrestricted, the wholesale prices changes with the manufacturer purchase quantity. The wholesale price of major supplier in single sourcing is lower than that in dual sourcing. The wholesale price of backup supplier in normal state is lower than that in disrupted state.

Let as define disruption proportion and delivery proportion. Major supplier may be partially interrupted or completely interrupted. Disruption proportion is defined as the ratio of the rest supply capacity to all the ordering quantity with partially interruption. In dual sourcing manufacturer assigns a backup supplier to a certain proportion of orders and this proportion is called by delivery proportion.

\subsection{Symbol Description}

$M:$ maximum market scale

$\Delta M$ : market scale shifting when disruption occurs

$D$ : realized demand, $D=M-k c$

$k$ : coefficient of price sensitivity, $k>0$

$\gamma:$ disruption probability of main supplier

$p$ : unit sales price of final product

$c_{m}^{1}$ : main supplier's unit wholesale price in singlesourcing

$c_{m}^{2}$ : main supplier's unit wholesale price in dual-sourcing

$c_{b}^{1}$ : backup supplier's unit wholesale price in normal state $c_{b}^{2}:$ backup supplier's unit wholesale price in disrupted state

$v$ : manufacturer's unit loss of unsatisfied demand

$\alpha$ : main supplier disruption proportion in disrupted state, $0 \leq \alpha \leq 1$

$\beta$ : backup supplier delivery allocation in dualsourcing, $0 \leq \beta \leq 1$

$c_{m}^{1}<c_{m}^{2}<c_{b}^{1}<c_{b}^{2}$

\section{SOURCING STRATEGY SELECTION BASED ON SUPPLY DISRUPTIONS}

The manufacturer has two sourcing methods to choose from, one is single sourcing and the other is dual souring. Different purchasing strategies have different prices, depicted in Table 1. We use the average of their wholesale prices to calculate the market demand in dual sourcing.

\subsection{The Benchmark with Single Sourcing and Dual Sourcing}

The expected profit function of the manufacturer with single sourcing, $E\left(\pi_{1}\right)$, is given by

$$
\begin{aligned}
& E\left(\pi_{1}\right)=(1-\gamma)\left(p-c_{m}^{1}\right)\left(M-k c_{m}^{1}\right)+\gamma \cdot\left[\alpha\left(M+\Delta M-k c_{m}^{1}\right) \cdot v+\right. \\
& \left.(1-\alpha)\left(p-c_{m}^{1}\right)\left(M-k c_{m}^{1}\right)\right]
\end{aligned}
$$

The expected profit function of the manufacturer with dual sourcing, $E\left(\pi_{2}\right)$, can be calculated as

$$
\begin{aligned}
E\left(\pi_{2}\right)= & (1-\gamma)\left(M-k \cdot \frac{c_{m}^{2}+c_{b}^{1}}{2}\right)\left[\left(p-c_{m}^{2}\right)-\beta\left(c_{b}^{1}-c_{m}^{2}\right)\right] \\
& +\gamma \cdot\left[\alpha(1-\beta)\left(p-c_{b}^{2}\right)\left(M-\frac{c_{m}^{2}+c_{b}^{1}}{2}\right)+(1-\alpha)(1-\beta)\right. \\
& \left(p-c_{m}^{2}\right)\left(M-\frac{c_{m}^{2}+c_{b}^{1}}{2}\right)+\beta\left(p-c_{b}^{1}\right)\left(M-k \frac{c_{m}^{2}+c_{b}^{1}}{2}\right)+ \\
& \left.\left(\Delta M-k \frac{c_{b}^{1}+c_{b}^{2}}{2}\right) \cdot v\right]
\end{aligned}
$$

When the backup supplier is the sole source, $\alpha=0, \beta=1$. The expected profit function of the manufacturer is given by

$$
\begin{aligned}
E\left(\pi_{3}\right)= & (1-\gamma) \cdot\left[\left(p-c_{b}^{1}\right)\left(M-k c_{b}^{1}\right)\right]+\gamma\left\{\left(p-c_{b}^{1}\right)\left(M-k c_{b}^{1}\right)+\right. \\
& {\left.\left[\left(M+\Delta M-k c_{b}^{2}\right)-\left(M-k c_{b}^{1}\right)\right] v\right\}=\left(p-c_{b}^{1}\right)\left(M-k c_{b}^{1}\right) } \\
& +\gamma\left[\left(M+\Delta M-k c_{b}^{2}\right)-\left(M-k c_{b}^{1}\right)\right] v
\end{aligned}
$$


When dual sourcing method outperforms the single sourcing with the main supplier as the sole source, $E\left(\pi_{2}\right)>E\left(\pi_{1}\right)$,

$(1-\gamma)\left(M-k \cdot \frac{c_{m}^{2}+c_{b}^{1}}{2}\right)\left[\left(p-c_{m}^{2}\right)-\beta\left(c_{b}^{1}-c_{m}^{2}\right)\right]+\gamma$.

$\left[\alpha(1-\beta)\left(p-c_{b}^{2}\right)\left(M-\frac{c_{m}^{2}+c_{b}^{1}}{2}\right)+(1-\alpha)(1-\beta)\left(p-c_{m}^{2}\right)\right.$

$\left(M-\frac{c_{m}^{2}+c_{b}^{1}}{2}\right)+\beta\left(p-c_{b}^{1}\right)\left(M-k \frac{c_{m}^{2}+c_{b}^{1}}{2}\right)+$

$\left.\left(\Delta M-k \frac{c_{b}^{1}+c_{b}^{2}}{2}\right) \cdot v\right]>(1-\gamma)\left(p-c_{m}^{1}\right)\left(M-k c_{m}^{1}\right)+\gamma$.

$\left[\alpha\left(M+\Delta M-k c_{m}^{1}\right) \cdot v+(1-\alpha)\left(p-c_{m}^{1}\right)\left(M-k c_{m}^{1}\right)\right]$

The expression can be written as,

$$
\begin{gathered}
\gamma>\gamma_{1}=\frac{\left(p-c_{m}^{1}\right)\left(M-k c_{m}^{1}\right)-\left(M-k \cdot \frac{c_{m}^{2}+c_{b}^{1}}{2}\right)\left[\left(p-c_{m}^{2}\right)-\beta\left(c_{b}^{1}-c_{m}^{2}\right)\right]}{\left(M-k \cdot \frac{c_{m}^{2}+c_{b}^{1}}{2}\right)(\beta-1)\left(\alpha c_{b}^{2}-\alpha c_{m}^{2}\right)-\left[\Delta M-k \frac{c_{b}^{1}+c_{b}^{2}}{2}+\right.} \\
\left.\alpha\left(M+\Delta M-k c_{m}^{1}\right)\right] v+\alpha\left(p-c_{m}^{1}\right)\left(M-k c_{m}^{1}\right)
\end{gathered}
$$

If $\gamma>\gamma_{1}$, then $E\left(\pi_{2}\right)>E\left(\pi_{1}\right)$. In this context, dual sourcing is better than single sourcing with the main supplier.

When the single sourcing with the backup supplier as the sole source dominates the dual sourcing, then $E\left(\pi_{3}\right)>E\left(\pi_{2}\right)$

$\left(p-c_{b}^{1}\right)\left(M-k c_{b}^{1}\right)+\gamma\left[\left(M+\Delta M-k c_{b}^{2}\right)-\left(M-k c_{b}^{1}\right)\right] v>$

$(1-\gamma)\left(M-k \cdot \frac{c_{m}^{2}+c_{b}^{1}}{2}\right)\left[\left(p-c_{m}^{2}\right)-\beta\left(c_{b}^{1}-c_{m}^{2}\right)\right]+\gamma \cdot[\alpha(1-\beta)$

$\left(p-c_{b}^{2}\right)\left(M-\frac{c_{m}^{2}+c_{b}^{1}}{2}\right)+(1-\alpha)(1-\beta)\left(p-c_{m}^{2}\right)\left(M-\frac{c_{m}^{2}+c_{b}^{1}}{2}\right)$

$\left.+\beta\left(p-c_{b}^{1}\right)\left(M-k \frac{c_{m}^{2}+c_{b}^{1}}{2}\right)+\left(\Delta M-k \frac{c_{b}^{1}+c_{b}^{2}}{2}\right) \cdot v\right]$

We can get the following expression,

$$
\begin{gathered}
\gamma \geq \gamma_{2}=\frac{\left(M-k \cdot \frac{c_{m}^{2}+c_{b}^{1}}{2}\right)\left[\left(p-c_{m}^{2}\right)-\beta\left(c_{b}^{1}-c_{m}^{2}\right)\right]-}{\left(M-k c_{b}^{1}\right)} \\
\left(-k \frac{c_{m}^{2}+c_{b}^{1}}{2}\right)\left[\alpha(1-\beta)\left(c_{b}^{2}-c_{m}^{2}\right)-\beta\left(p-c_{m}^{2}\right)\right]+ \\
2 \\
\left(-k c_{b}^{2}-k c_{b}^{1}\right) \cdot v
\end{gathered}
$$

The model of backup supplier as the sole source is superior to dual sourcing.

Therefore, we can choose the appropriate procurement strategies according to the disruption probability:

If $\gamma<\gamma_{1}$, then choose main supplier as the sole source.

If $\gamma_{1}<\gamma<\gamma_{2}$, then choose dual souring.
If $\gamma>\gamma_{2}$, then choose backup supplier as the sole source.

\subsection{Analysis of the Disruption Proportion $\alpha$}

The derivatives of $E\left(\pi_{1}\right)$ with respect to $\alpha$ is given by,

$$
\frac{\partial E\left(\pi_{1}\right)}{\partial \alpha}=\gamma\left(k c_{m}^{1}-M-\Delta M\right) v-\left(p-c_{m}^{1}\right)\left(M+\Delta M-k c_{m}^{1}\right)
$$

It is obvious of $\gamma>\frac{c_{m}^{1}-p}{v}$, so $\frac{\partial E\left(\pi_{1}\right)}{\partial \alpha}<0$. When disruption proportion increases in single sourcing, the manufacturer's expected profit decreases.

The derivatives of $E\left(\pi_{2}\right)$ with respect to $\alpha$ becomes,

$$
\begin{aligned}
& \frac{\partial E\left(\pi_{2}\right)}{\partial \alpha}=\gamma(1-\beta)\left(c_{m}^{2}-c_{b}^{2}\right)\left(M-k \frac{c_{m}^{2}+c_{b}^{1}}{2}\right) \\
& \text { If } \gamma(1-\beta)\left(c_{m}^{2}-c_{b}^{2}\right)\left(M-k \frac{c_{m}^{2}+c_{b}^{1}}{2}\right)<0 \text {, then } \frac{\partial E\left(\pi_{2}\right)}{\partial \alpha}<0 . \text { The }
\end{aligned}
$$

manufacturer's expected profit decreases in disruption proportion.

Because the disruption portion of main supplier is complementary by the backup supplier, backup supplier's unit wholesale price in disrupted state is higher than main supplier's unit wholesale price. The manufacturer's expected profit decreases with disruption proportion.

\subsection{Analysis of the Delivery Proportion $\beta$} by

The derivatives of $E\left(\pi_{2}\right)$ with respect to $\beta$ can be written

$$
\begin{aligned}
\frac{\partial E\left(\pi_{2}\right)}{\partial \beta}= & \left(c_{m}^{2}-c_{b}^{1}\right)\left(M-k \cdot \frac{c_{m}^{2}+c_{b}^{1}}{2}\right) \\
& +\gamma \alpha\left(c_{b}^{2}-c_{m}^{2}\right)\left(M-k \cdot \frac{c_{m}^{2}+c_{b}^{1}}{2}\right)
\end{aligned}
$$

Considering that $\alpha<\frac{c_{b}^{1}-c_{m}^{2}}{c_{b}^{2}-c_{m}^{2}}$, we can obtain that as $\gamma>\frac{c_{b}^{1}-c_{m}^{2}}{x\left(c_{b}^{2}-c_{m}^{2}\right)}$, then $\frac{\partial E\left(\pi_{2}\right)}{\partial \beta}>0$; as $\gamma<\frac{c_{b}^{1}-c_{m}^{2}}{x\left(c_{b}^{2}-c_{m}^{2}\right)}$, then $\frac{\partial E\left(\pi_{2}\right)}{\partial \beta}<0$

When the delivery allocation satisfies $\gamma_{1}<\gamma<\frac{c_{b}^{1}-c_{m}^{2}}{\alpha\left(c_{b}^{2}-c_{m}^{2}\right)}$, the manufacturer's expected profit decreases with delivery allocation in dual sourcing. When the delivery allocation satisfies $\frac{c_{b}^{1}-c_{m}^{2}}{\alpha\left(c_{b}^{2}-c_{m}^{2}\right)}<\gamma<\gamma_{2}$, the manufacturer's expected profit increases in delivery proportion. When the delivery allocation is greater than a critical value, the manufacturer increases order quantity from backup sourcing, although this leads to higher procurement costs, but the total expected profit is heightened. 
Table 2. Parameter assignments.

\begin{tabular}{|c|c|c|c|c|c|c|c|c|c|c|c|}
\hline Parameter & $c_{m}^{1}$ & $c_{m}^{2}$ & $c_{b}^{1}$ & $c_{b}^{2}$ & $v$ & $p$ & $M$ & $\Delta M$ & $K$ & $\beta$ & $\alpha$ \\
\hline Values & 3 & 4 & 5 & 7 & -2 & 10 & 100 & 35 & 5 & 0.3 & 0.8 \\
\hline
\end{tabular}

\section{ANALYSIS OF NUMERICAL EXAMPLE}

In this section, analyze the manufactures expected profit of adopting single sourcing and multiple sourcing, the impact of the disruption proportion of major supplier and delivery allocation of backup supplier on the manufacturer's procurement strategy. Parameter assignments are shown in Table 2 .

The horizontal axis is disruption probability of main supplier, the vertical axis is manufacturer's expected profit function, denoted by $E\left(\pi_{1}\right), E\left(\pi_{2}\right)$ and $E\left(\pi_{3}\right)$. With the increase of disruption risk, manufacturer's expected profit reduces in three sourcing. There have two values of the disruption probability for decision-making, $\gamma=0.25$ or $\gamma=0.74$. The value 0.25 is the intersection of $E\left(\pi_{1}\right)$ and $E\left(\pi_{2}\right)$, while the value 0.74 is the intersection of $E\left(\pi_{2}\right)$ and $E\left(\pi_{3}\right)$. If $\gamma<0.25$, then choose main supplier as the sole source. If $0.25<\gamma<0.74$, then choose dual souring. If $\gamma>0.74$, then choose backup supplier as the sole source (Fig. 1).

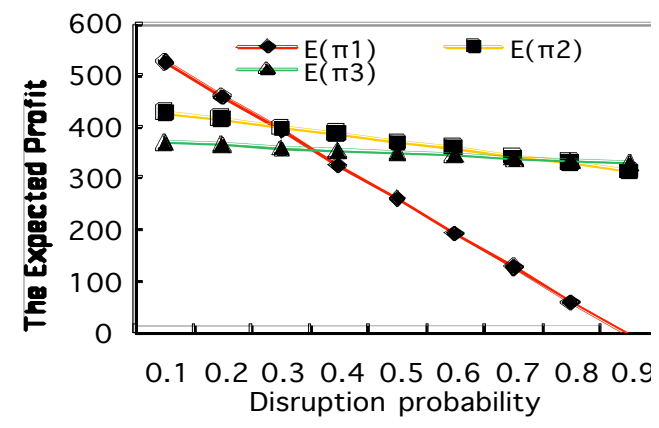

Fig. (1). Expected profits under different purchasing strategies.

The horizontal axis is disruption proportion of main supplier, the vertical axis is manufacturer's expected profit function in dual sourcing $E\left(\pi_{2}\right)$. We use numerical examples to show the sensitivity of manufacturer's expected profit in dual-sourcing to the two key parameters, $(\gamma, \alpha)$, which $\gamma$ takes values from 0.1 to 0.6 with the increment $0.1, \alpha$ takes values from 0.1 to 0.9 with the increment 0.1 . These points are plotted in Fig. (2). It shows that manufacturer's expected profit decreases in disruption proportion, and with the increase of disruption probability, the trend of decline gets faster. Because the disruption partial of main supplier is complementary by the backup supplier, backup supplier's unit wholesale price in disrupted state is higher than main supplier's. Thus manufacturer expected profit decreases in disruption proportion (Fig. 2).

The horizontal axis is distribution proportion of backup supplier, while the vertical axis is manufacturer's expected profit function in dual sourcing. We use numerical examples to show the sensitivity of manufacturer's expected profit in dual-sourcing to the two key parameters $(\gamma, \beta)$. Then can calculated the manufacturer's expected profit against $(\gamma, \beta)$, where $\gamma$ takes values from 0.3 to 0.7 , and $\beta$ takes values from 0.1 to 0.9 with the increment 0.1 . These points are plotted in Fig. (3). It shows the manufacturer's expected profit under different delivery allocation and different disruption probability in dual sourcing. When $\gamma<0.55$, the manufacturer's expected profit decreases with delivery allocation. When $\gamma=0.55$, the manufacturer's expected profit remains unchanged even if the value of $\beta$ varies. When $\gamma>0.55$, the expected profit increases with delivery allocation. When disruption probability low the threshold, considering the backup supplier wholesale price higher than main suppliers of wholesale price, increasing the distribution proportion of backup supplier will lower the expected profit. When disruption probability exceed the threshold, considering the backup supplier wholesale price in disrupted state higher than other in normal state, then the manufacturer can increase the distribution proportion to increase expected profit (Fig. 3).

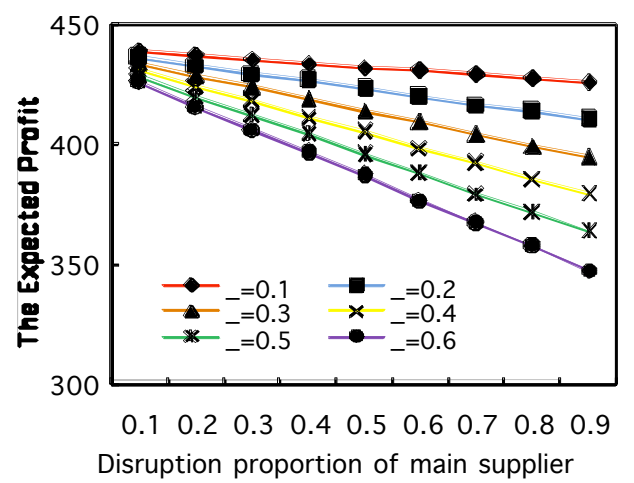

Fig. (2). Expected profits under different disruption proportion in dual sourcing.

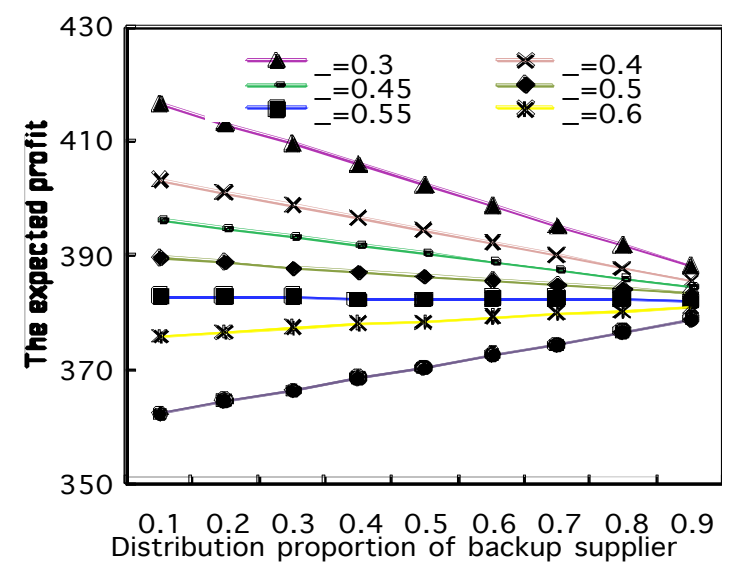

Fig. (3). Expected profits under different delivery allocation in dual sourcing. 


\section{CONCLUSION}

We study emergency procurement model based on incomplete supply disruptions, and analyze the manufacturer's expected profit of single sourcing procurement strategy and multiple sourcing procurement strategy under different risk of disruption. Furthermore, we argue the impact of the disruption proportion of major supplier and delivery allocation of backup supplier on the manufacturer's procurement strategy, and find out the best procurement strategy that the manufacturers should choose to maximize expected profit in different situations. We find that manufacturer's expected profit decreases in disruption proportion, and sharply reduced with disruption probability. As the disruption probability is below the critical value, the expected profit decreases with delivery allocation. As the disruption probability is higher than the critical value, the expected profit increases with delivery allocation.

There are other avenues to extend this article. First, based on the manufacturer's profit, we can examine the entire supply chain profits. Second, we can investigate limited supplier's capacity. Finally, our framework can be extended to consider more qualified suppliers.

\section{CONFLICT OF INTEREST}

The authors confirm that this article content has no conflict of interest.

\section{ACKNOWLEDGEMENTS}

This work is supported by the National Social Science Fund of China (No. 12CGL042), the National Natural Science Foundation of China (No. 71372122, 71402158 and 71271039).

\section{REFERENCES}

[1] C. Blame, and M. Henke, "Supply chain risk: a handbook of assessment, management and performance," Springer-Verlag: New York, USA, 2009.
[2] O. Tang, and S. Nurmaya, "Identifying risk issues and research advancements in supply chain risk management," International Journal of Production Economics, vol. 1, pp. 25-34, 2011.

[3] G. Burke, J. Carrillo, and A. Vakharia, "Single versus multiple supplier sourcing strategies," European Journal of Operational Research, vol. 1, pp. 95-112, 2007.

[4] W. Wang, "The Effect on Optimal Ordering Quantity Under Disruption Risk of Two Supplier," PhD Thesis of Taiwan University, 2011

[5] H.Yu, and A. Z. Zeng, "Single or dual sourcing: decision-making in the presence of supply chain disruption risks," OMEGAInternational Journal of Management Science, vol. 4, pp. 788-800, 2009.

[6] K. Chen, and P. Zhuang, "Disruption management for a dominant retailer with constant demand-stimulating service cost," Computers \& Industrial Engineering, vol. 4, pp. 936-946, 2011.

[7] F. J. Sting, and A. Huchzermeier, "Dual sourcing: responsive hedging against correlated supply and demand uncertainty," Naval Research Logistics, vol. 1, pp. 69-89, 2012.

[8] X, Li, J. Ji, and S. Wang, "Coordination and optimization of dual sourcing under supply disruptions," Journal of Industrial Engineering and Engineering Management, vol. 3, pp. 141-147, 2014.

[9] B. Tomlin, and Y. Wang, "On the value of mix flexibility and dual sourcing in unreliable newsvendor networks," Manufacturing \& Service Operations Management, vol. 1, pp. 37-57, 2005.

[10] J. Hou, and A. Z. Zeng, "Coordination with a backup supplier through buy-back contract under supply disruption," Transportation Research Part E, vol. 5, pp. 340-347, 2010.

[11] W. Zhang, "Analysis on supply emergency management based on dual source," Journal of Shanghai Jiao Tong University, vol. 3, pp. 454-458, 2013.

[12] X. Li, J. Ji, and S. Wang, "Performance analysis of backup supplier for supply disruptions with stochastic demand," Operations Research and Management Science, vol. 4, pp. 42-49, 2013.

[13] J. Chen, X. Zhao, and Y. Zhou, "A periodic-review inventory system with a capacitated backup supplier for mitigating supply disruptions," European Journal of Operational Research, vol. 2, pp. 312-323, 2012.

[14] V. Babich, "Vulnerable options in supply chains: effects of supplier competition," Naval Research Logistics, vol. 7, pp. 656$673,2006$. 